\section{Obstructive sleep apnoea, insulin resistance and sleepiness}

\author{
Mary S M Ip
}

Our understanding of obstructive sleep apnoea (OSA) has advanced much beyond that of the condition being a noisy breathing abnormality during sleep which classically presents with daytime sleepiness, to a condition with numerous potentially significant downstream sequelae and multisystem manifestations. The relationship between OSA and metabolic dysfunction is a moving target. Cross sectional studies have provided suggestive evidence for an independent relationship between OSA and adverse glucose metabolism, while supportive longitudinal data and interventional data from randomised controlled trials are still lacking. ${ }^{1}$ Recently, data from the Sleep Heart Health Study again confirmed the independent association between sleep disordered breathing and impaired glucose metabolism in normal weight and overweight/obese individuals. ${ }^{2}$ In this issue of Thorax, the study of Barceló and colleagues ${ }^{3}$ suggested that the presence/absence of daytime sleepiness determined the presence/absence of increased insulin resistance in OSA, and that continuous positive airway pressure (CPAP) treatment decreased insulin resistance in sleepy subjects but had no effect in nonsleepy subjects (see page 946). Their findings showed a pattern analogous to the previously reported phenomenon regarding OSA and elevated blood pressure, whereby the severity of daytime sleepiness in OSA predicted a blood pressure lowering response to CPAP treatment. ${ }^{45}$

Excessive daytime sleepiness has always been considered as one of the cardinal symptoms of OSA, and it is usually improved by effective treatment of OSA. However, it has also been well recognised that excessive daytime sleepiness is not universally present in OSA subjects, and that despite similar severity of sleep disordered breathing based on the apnoea-hypopnoea index (AHI), the degree of daytime sleepiness in individuals may be quite dissimilar. The aetiology of daytime sleepiness in OSA in terms of nocturnal sleep components has been

Correspondence to: Professor Mary S M Ip, Department of Medicine, The University of Hong Kong, Queen Mary Hospital, Pokfulam, Hong Kong SAR, China; msmip@hkucc.hku.hk explored, with variable findings for the relative contributions of sleep fragmentation, hypoxaemia and other parameters. ${ }^{6-9}$ In a multicentre cohort study, subjects who had daytime sleepiness demonstrated longer sleep duration and increased slow wave sleep compared with non-sleepy subjects, and sleepiness was not primarily determined by sleep apnoea and sleep disruption. ${ }^{9}$ Putting aside the issue of measurements of sleepiness and sleep apnoea, which in themselves are not without controversy, the discrepancy between the severity of sleep apnoea and that of daytime sleepiness serve to underscore that the ultimate expression of sleepiness, or for that matter metabolic dysfunction or any other sequelae in OSA, is multifactorial and subject to various genetic and environmental influences, including lifestyle behaviour. ${ }^{10}{ }^{11}$ In this context, the finding of an independent association of daytime sleepiness and increased insulin resistance in OSA suggests the intriguing possibility of some links among various adverse sequelae of OSA.

A number of epidemiological studies have reported that short sleep duration or sleep disturbances, which are potential causes of sleep loss and daytime sleepiness, were associated with increased population study found that daytime sleepiness was more strongly associated with depression and metabolic factors (obesity/diabetes) than with sleep apnoea or objective sleep disruption per se. ${ }^{9}$ Another population based study showed that daytime sleepiness either with or without snoring was a risk factor for diabetes in women. ${ }^{13}$ In a small sample of non-diabetic women with chronic obstructive pulmonary disease, sleepiness was found to be more associated with higher levels of fasting insulin rather than short and fragmented sleep. ${ }^{14}$

The mechanistic basis of excessive daytime sleepiness or that of metabolic dysfunction in OSA has not been well elucidated, and obviously even less so for their potential linkage. Obesity per se has been indicated as a cause of excessive daytime sleepiness, with a presumptive diabetic risks. ${ }^{12}$ A randomised general physiological basis that fat tissue is a source of low grade fatigue causing cytokines or hormones. ${ }^{15}$ The use of body mass index (BMI) matched OSA groups in Barceló's study ${ }^{3}$ was intended to obviate the confounding effect of obesity on either sleepiness or insulin resistance, but the need remains for more careful examination of the role of visceral obesity. Furthermore, the similar degree of insulin resistance in the non-sleepy OSA compared with healthy controls with a significantly lower BMI was somewhat unexpected. The triad of OSA, daytime sleepiness and insulin resistance may represent a common pathogenetic mechanism for both the propensity to sleep and to adverse glucose metabolism, such as through certain proinflammatory cytokines; or one sequelae of OSA may precede and predispose to the other, such as sleepiness predisposing to physical inactivity which then promotes insulin resistance or vice versa with insulin resistance involved in somnogenesis; or the sleepy phenotype in itself, regardless of the presence or absence of OSA, is associated with insulin resistance. Any of these pathways would be subjected to further modulation by endogenous and exogenous factors, and interactions among themselves. ${ }^{10} 111516$ Putative candidates include oxidative stress, inflammation and neurohumeral responses, and mediators such as interleukin 6, tumour necrosis factor, cortisol, hypocretin, grhelin, leptin, catecholamines $^{12}$ 15-17 $^{17}$ and, in Barceló's study, insulin-like growth factor $1 .{ }^{3}$

Barceló's study ${ }^{3}$ also observed a greater oxygen desaturation in the sleepy OSA group, despite similar AHI and BMI, compared with the non-sleepy OSA group. Without further information of the "respiratory reserve" in these otherwise healthy subjects, in particular the effect of abdominal obesity or smoking, one cannot definitively exclude the contribution of these factors towards sleep hypoxaemia. However, given the similar BMI and proportion of smokers in both groups, the contribution would be expected to be small even if present. It is tempting to surmise that greater hypoxaemia due to sleep apnoea is the fundamental physiological trigger of insulin resistance or sleepiness, or both. Previous clinical and epidemiological studies have demonstrated an independent association between insulin resistance and sleep hypoxaemia in OSA. ${ }^{18} 19$ Animal studies have also demonstrated that intermittent hypoxia may induce insulin resistance. ${ }^{2021}$ On the other hand, intermittent hypoxia 
in animal studies has been shown to result in neuronal damage in wake promoting structures or disturbance in sleep architecture. $^{22} 23$ Notwithstanding, one can also look at the issue of sleep hypoxaemia and daytime sleepiness from other angles. Why was desaturation more severe in the sleepy group despite similar $\mathrm{AHI}$ ? Can the daytime sleepiness in those OSA individuals be a reflection of an overall propensity to sleep ${ }^{911}$ with diminished ventilatory response during nocturnal sleep, hence greater oxygen desaturation? Apart from hypoxaemia, is there any role for hypercarbia, the parameter in oblivion which is expected to be fluctuating alongside oxygen saturation?

Barceló's article $e^{3}$ has raised many more questions than it can answer. While researchers speculate on, and attempt to delineate, the complex relationships between OSA and its potential metabolic and neurobehavioural sequelae, it is important to confirm the findings of this work with further clinical trials. Furthermore, these two groups of subjects with similar severe OSA by AHI criteria and similar BMI, yet widely divergent degrees of daytime sleepiness, were probably highly selected for the purpose of addressing the authors' stated hypothesis. In our daily encounter, subjects would have a wide spectrum of severity of OSA, obesity and sleepiness. Given the numerous permutations in phenotypes and their underlying pathophysiological interactions, what demarcates clearly in the study sample may have very different consequences in the wider population of OSA subjects. This is perhaps the challenge of researchto search, and research.

Competing interests: None declared.

Thorax 2008;63:939-940. doi:10.1136/thx.2008.099689

\section{REFERENCES}

1. Tasali E, Ip MSM. Obstructive sleep apnea and metabolic syndrome: alterations in glucose metabolism and inflammation. Proc Am Thorac Soc 2008;5:207-17.

2. Seicean S, Kirchner HL, Gottlieb DJ, et al. Sleep disordered brathing and impaired glucose metabolism in normal-weight and overweight/obese individuals. Diabetes Care 2008:31:1001-6.

3. Barceló A, Barbé $F$, de la Peña $M$, et al. Insulin resistance and daytime sleepiness in patients with sleep apnea. Thorax 2008;63:946-50.

4. Barbé F, Mayoralas LR, Duran J, et al. Treatment with continuous positive airway pressure is not effective in patients with sleep apnea but no daytime sleepiness, a randomized, controlled trial. Ann Inter Med 2001;134:1015-23.

5. Robinson GV, Langford BA, Smith DM, et al. Predictors of blood pressure fall with continuous positive airway pressure (CPAP) treatment of obstructive sleep apnoea (OSA). Thorax 2008 Apr 3 (Epub ahead of print).

6. Guilleminault C, Partinen M, Quera-Salva MA, et al Determinants of daytime sleepiness in obstructive sleep apnea. Chest 1988;94:32-7.

7. Punjabi NM, O'Hearn DJ, Neubauer DN, et al. Modeling hypersomnia in sleep disordered breathing Am J Respir Crit Care Med 1999;159:1703-9.

8. Bixler EO, Vgontzas AN, Lin HM, et al. Excessive daytime sleepiness in a general population sample: the role of sleep apnea, age, obesity, diabetes and depression. J Clin Endocrinol Metab 2005:90:4510-15.

9. Roure N, Gomez S, Mediano 0, et al. Daytime sleepiness and polysomnography in obstructive sleep apnea patients. Sleep Med 2008 May 13 (Epub ahead of print).

10. Gozal D, Kheirandish L. Oxidant stress and inflammation in the snoring child: confluent pathways to upper airway pathogenesis and end-organ morbidity. Sleep Med Rev 2006;10:83-96.
11. Gottlieb DJ, O'Connor GT, Wil JB. Genome-wide association of sleep and circadian phenotypes. BMC Med Genet 2007;8(Suppl 1):59.

12. Ip M, Mokhlesi B. Sleep and glucose intolerance/ diabetes mellitus. Sleep Med Clin 2007;2:19.

13. Lindberg $\mathbf{E}$, Berne $\mathrm{C}$, Franklin KA, et al. Snoring and daytime sleepiness as risk factors for hypertension and diabetes in women-a population-based study. Respir Med 2007;101:1283-90.

14. Saaresranta T, Irjala K, Aittokallio T, et al. Sleep quality, daytime sleepiness and fasting insulin levels in women with chronic obstructive pulmonary disease. Respir Med 2005;99:856-63.

15. Vgontzas AN, Bixler EO, Chrousos GP. Obesityrelated sleepiness and fatigue: The role of the stress system and cytokines. Ann NY Acad Sci 2006;1083:329-44.

16. Knutson KL, Spiegel K, Penev P, et al. The metabolic consequences of sleep deprivation. Sleep Med Rev 2007; 11:163-78

17. de la Pena BM, Serpero LD, Barcelo A, et al. Inflammatory proteins in patients with obstructive sleep apnea with and without daytime sleepiness. Sleep Breath 2007:11:177-85.

18. Ip MSM, Lam B, Ng MMT, et al. Insulin resistance is independently associated with obstructive sleep apnea. Am J Respir Crit Care Med 2002;165:670-6.

19. Punjabi NM, Shahar E, Redline S, et al, for the Sleep Heart Health Study Investigators. Sleep disordered breathing, glucose intolerance and insulin reistance. Am J Epidemiol 2004;160:521-30.

20. Polotsky VY, Li J, Punjabi NM, et al. Intermittent hypoxia increases insulin resistance in genetically obese mice. J Physiol 2003;552:253-64.

21. Liyori $\mathbf{N}$, Alonso LC, Li J, et al. Intermittent hypoxia causes insulin resistance in lean mice independent of autonomic activity. Am J Respir Crit Care Med 2007:175:851-7.

22. Veasey SC, Davis CW, Fenik P, et al. Long-term intermittent hypoxia in mice: protracted hypersomnolence with oxidative injury to sleep-wake brain regions. Sleep 2004:27:194-201.

23. Polotsky VY, Rubin AE, Balbir A, et al. Intermittent hypoxia causes REM sleep deficits and decreases EEG delta power in NREM sleep in the C57BL/6J mouse. Sleep Med 2006;7:7-16.

\section{Antibiotics at COPD exacerbations: the debate continues}

\section{Jadwiga A Wedzicha}

The course of chronic obstructive pulmonary disease (COPD) is affected by the presence of exacerbations that are episodes of worsening of respiratory symptoms commonly triggered by airway

Correspondence to: Professor Jadwiga A Wedzicha, Academic Unit of Respiratory Medicine, Royal Free and University College Medical School, Rowland Hill Street, Hampstead, London NW3 2PF, UK; J.A.Wedzicha@ medsch.ucl.ac.uk infections, including respiratory viruses and airway bacteria. ${ }^{1}$ COPD exacerbations have important adverse effects on health status ${ }^{2}$ and mortality ${ }^{3}$ and affect the course of the disease. ${ }^{4}$ Thus there is considerable interest in the effectiveness of interventions used both to treat exacerbations and prevent further events.

Exacerbations are usually treated with oral corticosteroids and/or antibiotics depending on exacerbation severity and the nature of the symptoms. There is now considerable evidence for benefit of a course of oral corticosteroids at exacerba$\operatorname{tion}^{56}$ and also for antibiotics when two of the three symptoms of increased dyspnoea, sputum volume and purulence are present. ${ }^{7}$ However, there is still some controversy concerning the role of antibiotics at COPD exacerbation, especially in studies performed in primary care ${ }^{8}$ where generally patients with milder disease have been recruited. There is now clear documentation of the involvement of bacteria at exacerbations with increased bacterial detection and load, ${ }^{9}$ while bacterial strain changes have also been associated with development of exacerbations. ${ }^{10}$ Eradication of bacteria in the airways with antibiotics prescribed at exacerbation has been linked to exacerbation recovery and reduction in airway inflammatory markers. ${ }^{11}$ Some of the controversy regarding 